\title{
Tecnura
}

\section{Externalidades de las emisiones del transporte público en Tepic, México: cambio climático y sustentabilidad}

\section{Externalities of the public transportation emissions in tepic, mexico: climate change and sustainability}

\author{
Sylvia Lorena Serafín González ${ }^{1}$
}

Fecha de recepción: 9 de mayo de 2019

Fecha de aceptación: 23 de agosto de 2019

Como citar: Serafín G., S.L. (2019). Externalidades de las emisiones del transporte público en Tepic, México: cambio climático y sustentabilidad. Tecnura, 23(62), 34-44. https://doi.org/10.14483/22487638.15455

\section{Resumen}

Contexto: Los procesos antrópicos relacionados con la movilidad urbana han colocado en el tema del transporte público en la agenda de la mayoría de países. Efectivamente, comparado con el vehículo privado, este representa la forma más económica y sustentable de ejercer la movilidad cotidiana. Sin embargo, quienes utilizan el proceso de combustión interna, a través de derivados del petróleo, emiten gases de efecto invernadero, y por tanto, esta forma de movilidad motorizada se ha vuelto el mayor contribuyente al cambio climático global. Bajo esta premisa, el objetivo de este artículo consiste en analizar las externalidades de las emisiones de gases de efecto invernadero en el sector transporte público de autobuses en la ciudad de Tepic, México.

Método: La investigación utiliza datos proporcionados por la Secretaría del Medio Ambiente de Nayarit, a partir de los cuales se estimaron las emisiones de gases de efecto invernadero del transporte público utilizando la metodología de 1996 avalada por el Panel Intergubernamental del Cambio Climático de las Naciones Unidas. En este sentido, de acuerdo con esta metodología, que permite estimar el consumo de combustibles se obtuvo el recorrido anual por tipo de vehículo, así como su rendimiento vehicular.

Resultados: Las emisiones de gases de efecto invernado en la ciudad de Tepic, México, se aproximaron a las 192.000 toneladas emitidas por tan solo por transporte público de autobuses. Aunque el otorgamiento de concesiones se ha venido dando de manera desordenada y arbitraria por parte de los diferentes gobiernos, fue posible prospectar un escenario de emisión al 2020.

Conclusiones: El transporte público de autobuses en la ciudad de Tepic enfrenta un acelerado crecimiento, provocado por la expansión urbano-habitacional desordenada. Este sistema se ha convertido en uno de los principales emisores de gases de efecto invernadero, que lo colocan como tema central para la implementación de políticas públicas de transporte, adoptando iniciativas y mecanismos tendientes a reducir los niveles de emisiones, reducir externalidades y contribuir con la sustentabilidad y mitigación del cambio climático global.

Palabras clave: emisiones, cambio climático, susten-tabilidad, transporte público, México .

1 Licenciada en Contaduría, maestra en Derecho Económico Local, doctora en Geografía y Ordenación Territorial. Profesor titular, Universidad Politécnica de Nayarit. Tepic, México. Contacto: sylvia.serafin.gonzalez@upnay.edu.mx 


\section{Abstract}

Context: Anthropic processes related to urban mobility have placed the issue of public transportation on the agenda of most countries in the world. In fact, compared to the private vehicle, it represents the most economical and sustainable way of exercising daily mobility. However, who use the internal combustion process, through petroleum derivatives, emit greenhouse gases and, therefore, this form of motoring mobility has become the biggest contributor to global climate change. Under this premise, the aim of this article is to analyze the externalities of greenhouse gas emissions in the public transportation of buses in the city of Tepic, Mexico.

Method: The research uses data provided by the $\mathrm{Na}-$ yarit Ministry of Environment; from which greenhouse gas emissions from public transportation were estimated using the 1996 methodology approved by the United Nations Intergovernmental Panel on Climate Change. In this sense, according to this methodology, which allows to estimate fuel consumption, the annual route was obtained by type of vehicle, as well as the vehicle's performance.

Results: The greenhouse gas emissions in the city of Tepic, Mexico, approached 192 thousand tons emitted only by public transportation by bus. Although the granting of concessions has been carried out in a disorderly and arbitrary manner by the different governments, it was possible to provide an emission scenario for 2020.

Conclusions: Public bus transportation in the city of Tepic faces accelerated growth, caused by the disorderly expansion of urban housing. This system has become one of the main emitters of greenhouse gases, which makes it a central issue for the implementation of public transportation policies, adopting initiatives and mechanisms to reduce emission levels, reduce externalities and contribute to the sustainability and mitigation of global climate change.

Keywords: Emissions, climate change, sustainability, public transportation, Mexico.

\section{INTRODUCCIÓN}

El sector transporte es el que más participa en la emisión de gases de efecto invernadero (GEI). Este trabajo, presenta un panorama general del consumo de energía y emisiones que produce el transporte público de la ciudad de Tepic, México; igualmente, prospecta un posible escenario en función de la tendencia de crecimiento desordenado. En este sentido, uno de los problemas que afectan de manera importante la ciudad de Tepic tiene que ver con el transporte público, el cual ha crecido sin una planeación formal de una política pública integral de las diferentes autoridades de gobierno. En ese sentido, entropías ocasionadas por la congestión vial, deterioro en infraestructura por intemperismo y accidentalidad asociada al transporte se han convertido en parte de la vida cotidiana de los residentes urbanos. De ahí, la necesidad de implementar "medidas preventivas y correctivas que supriman la accidentalidad, la morbilidad y la mortalidad en la metrópolis" (García, González y Asprilla, 2018, p. 52). Sobre todo, porque la movilidad motorizada no solo perturba al sistema, sino a sus subsistemas y al ecosistema en general. Además, en relación de la variable tiempo de la clásica ecuación $v=d / t$, la introducción indiscriminada de nuevos vehículos al sistema vial, implica la generación de entropías vinculadas con el aumento del tiempo en los desplazamientos cotidianos; toda vez, que los incrementos de velocidad están regulados por la autoridad y la distancia geométrica asume una posición compleja de modificar (González, 2018a, p. 172).

Ciertamente, Tepic se ubica como una ciudad de menor magnitud poblacional respecto a las grandes metrópolis de México, como la Ciudad de México, y las metrópolis de Guadalajara y Monterrey. En este sentido, todavía no enfrenta de manera significativa los efectos de la contaminación ambiental por 
emisiones de gases provenientes de la movilidad motorizada. Sin embargo, esta situación ha ocasionado que se carezca de información relevante para la determinación de los niveles de contaminación de aire, y una omisión por parte de la autoridad competente en materia de base de datos que proporcione parámetros e indicadores de medición. En suma, se adolece de un monitoreo ambiental.

En este contexto, ante el incremento exponencial de la población, la degradación de los recursos no renovables, la irracionalidad en el sobreconsumo de fuentes energéticas fósiles, la obsolescencia tecnológica programada, la contaminación de los recursos renovables, entre otras entropías urbanas, se ha iniciado de una nueva era para la especie humana, dependiente cada vez más de la forma de vivir, habitar y transportarse en el territorio. Aquí, los impactos ambientales son "resultado de los procesos de industrialización y urbanización. Los daños ecológicos son síntomas que se hacen visibles años después de que iniciara su impacto, como efectos invisibles de acciones específicas" (Alfie, 2007, p. 217).

El proceso del habitar está en función del incremento poblacional y la movilidad. Es decir, a mayores incrementos demográficos le sigue un incremento habitacional necesario para satisfacer la demanda. Esta demanda de vivienda presiona al consumo de suelo. Asimismo, la movilidad se induce en función de la ubicación geográfica y la distancia. Así, a mayor población, mayor vivienda y mayor movilidad. No obstante, como el espacio es limitado, la movilidad de índole motorizada genera entropía en determinados horarios, "debido a la afluencia de personas que intentan desplazarse diariamente desde sus orígenes a sus destinos, y provocan conflictos viales durante las denominadas horas pico" (Arizaga, González y Asprilla, 2019, p. 14). En este sentido, el desarrollo de nuevas tecnologías tendientes a satisfacer las necesidades de la población que demanda mejorar su calidad de vida compromete la sustentabilidad de los recursos para las futuras generaciones. En suma, el crecimiento demográfico y habitacional desmedido mostrado en la figura 1 ha marcado una tendencia al consumo de mayores recursos territoriales que proveen los insumos necesarios en los procesos de transformación.

En función de lo anterior, la Organización de las Naciones Unidas (ONU) estableció una definición del cambio climático: "[es] un cambio de clima atribuido directa o indirectamente a la actividad humana que altera la composición de la atmósfera mundial y que se suma a la variabilidad natural del clima observada durante periodos de tiempo comparables" (ONU, 2018). Asimismo, de acuerdo con el reporte proporcionado por el Panel Intergubernamental del Cambio Climático (IPCC, 2017):

a. Las concentraciones atmosféricas de dióxido de carbono, metano y óxido nitroso mundiales han aumentado, sensiblemente, como resultado tanto del cambio en las actividades humanas como el aumento de la población. En la actualidad, se han superado los valores preindustriales determinados en muestras de testigos de hielo que datan de cientos de años. Este aumento de la concentración de dióxido de carbono a nivel mundial se debe principalmente al uso de combustibles fósiles y a los cambios sin control que se han dado en la mayoría de los países, y se relaciona con el uso del suelo, por cuanto el metano y el óxido nitroso se deben a la agricultura.

b. El calentamiento del clima es inequívoco; las mediciones marcan incrementos en las temperaturas medias tanto del aire como de los océanos. Igualmente, se derrite el hielo y la nieve de los polos, y ha subido la elevación del nivel del mar.

c. Tanto en regiones, países y continentes se observan cambios climáticos a largo plazo. Estos incluyen cambios en las temperaturas, en la cantidad de precipitaciones pluviales y en las condiciones climáticas extremas: sequías, fuertes lluvias, fuerte calor y la intensidad tanto de ciclones como de huracanes.

d. Se proyecta un calentamiento de cerca de $0,2{ }^{\circ} \mathrm{C}$ en las próximas dos décadas por emisiones de los escenarios del IEEE desarrollados por 
Nakicenovic (2000), que son utilizados como base para realizar las proyecciones climáticas.

e. Las emisiones de gases de efecto invernadero se han incrementado a diferentes tasas, debido a las emisiones directas del transporte y de la industria, y al cambio en los usos. El sector de la construcción también ha contribuido por el alto consumo de electricidad que demanda.

Sobre esto último, es preciso mencionar que según datos de la Secretaría del Medio Ambiente y Recursos Naturales de México (Semarnat, 2018), el transporte es el sector que más contribuye a las emisiones de GEI y el que presenta mayor crecimiento. En este entendido, analizar sus tendencias es indispensable para estimar el consumo de derivados de petróleo y mitigar las emisiones de GEI.

En el Protocolo de Kioto de la Convención Marco Climático de las Naciones Unidas, y donde México firmó los acuerdos internacionales en materia de reducción de emisiones de GEI, se establece el mecanismo de desarrollo limpio (MDL) como un instrumento de intercambio de emisiones. Es decir, este permitiría obtener recursos económicos mediante la venta de reducciones de emisiones de GEI. Sobre todo, cuando se sabe que el sector transporte es el mayor consumidor de combustibles fósiles en México tal y como se muestra en la tabla 1. Además, este aumento acelerado de los derivados del petróleo (gasolina y diésel) que utiliza el transporte aunado a la carente inversión en la refinación de petróleo repercuten también la importación de combustibles.
De acuerdo con la tabla anterior, el autotransporte es el mayor consumidor de combustible en el país. Este se subdivide en dos generalidades: transporte interurbano y transporte urbano. Ambos pueden destinarse a la carga de productos $y$ mercancías, o de pasajeros. En el caso de Tepic, los indicadores de actividad y eficiencia del sector transporte circunscriben:

a. El número de vehículos por tipo o modo. Esto se refiere al total de vehículos en circulación para determinado año y tipo; en otros términos, el tipo hace alusión al tamaño de vehículo o de autobús para el transporte de carga.

b. El número de kilómetros que recorre cada vehículo en un año.

c. La relación vehículo-kilómetro (V-K). Resulta de multiplicar la cantidad de vehículos de determinado tipo, por su distancia anual promedio recorrida en kilómetros. De manera que el indicador V-K para el total de las flotilla, se obtiene de hacer la suma de todos los vehículos de cada tipo de vehículo.

d. La relación tonelada-kilómetro (T-K). Muestra el resultado de multiplicar el indicador $\mathrm{V}-\mathrm{K}$ de cada tipo de transporte para carga, por el promedio de toneladas que lleva cada vehículo por viaje.

e. La relación pasajero-kilómetro (P-K). Indica el resultado de multiplicar la relación $\mathrm{V}$-K por el promedio de pasajeros que lleva cada vehículo por viaje. Ello se hace por cada tipo de vehículo para transporte de pasajeros.

f. El rendimiento vehicular. Muestra la cantidad de combustible que consume un vehículo por kilómetro recorrido.

Tabla 1. Consumo total de energía (petajulios)

\begin{tabular}{lcrr}
\hline & $\mathbf{2 0 1 6}$ & $\mathbf{2 0 1 7}$ & Variación $\%$ \\
\hline Consumo energético total & 4609,89 & 4735,71 & 2,73 \\
\hline Transporte & 2245,25 & 2283,98 & 1,73 \\
\hline Industrial & 1298,08 & 1363,43 & 5,03 \\
\hline Residencial, comercial y público & 921,25 & 928,25 & 0,76 \\
\hline Agropecuario & 145,32 & 160,06 & 10,14 \\
\hline
\end{tabular}

Fuente: elaboración propia con datos de Sener (2018). 
El incremento en el consumo de energía en sector transporte en el año 2017 respecto al año 2016, se debe principalmente al aumento del parque vehicular del autotransporte en detrimento de otros sistemas, como el ferroviario y el eléctrico (ver tabla 2).

En este contexto, con la información disponible en la tabla 3, los vehículos registrados de acuerdo con el Banco de Información Económica del INEGI (2017), entre el 2000 y el 2017 se tuvo un incremento en el número de automóviles del $304 \%$, autobuses en $198 \%$, camiones de carga $222 \%$ y motocicletas $1224 \%$. Esto evidencia que el parque vehicular repercute en el aumento del consumo de combustible del sector transporte. Igualmente, resalta el aumento desproporcional del transporte tipo motocicleta, el cual ha invadido prácticamente todas las ciudades del país; no obstante, es el que menores vías apropiadas tiene para su circulación y convierten en un riesgo tanto para el motociclista como para los otros modos de transporte (autobuses y vehículos privados) en

Tabla 2. Consumo de energía del sector transporte (petajulios)

\begin{tabular}{lrrr}
\hline & \multicolumn{1}{c}{$\mathbf{2 0 1 6}$} & \multicolumn{1}{c}{$\mathbf{2 0 1 7}$} & Variación \% \\
\hline Transporte & 2245,25 & 2283,98 & 1,73 \\
\hline Autotransporte & $20.170,33$ & 2100,39 & 1,45 \\
\hline Aéreo & 114,29 & 115,23 & 0,82 \\
\hline Marítimo & 29,96 & 35,73 & 19,23 \\
\hline Ferroviario & 26,52 & 28,75 & 8,39 \\
\hline Eléctrico & 4,14 & 3,88 & $-6,28$ \\
\hline
\end{tabular}

Fuente: elaboración propia con datos de Sener (2018).

Tabla 3. Vehículos de motor registrados, 2000-2017

\begin{tabular}{lccccc}
\hline Año & Total & Automóviles & $\begin{array}{c}\text { Autobuses de } \\
\text { Pasajeros }\end{array}$ & $\begin{array}{c}\text { Camiones de } \\
\text { Carga }\end{array}$ & Motocicletas \\
\hline 2000 & 15.611 .916 & 10.176 .179 & 202.396 & 4.939 .417 & 293.924 \\
\hline 2001 & 17.300 .530 & 11.351 .982 & 273.536 & 5.394 .206 & 280.806 \\
\hline 2002 & 18.784 .594 & 12.254 .910 & 299.365 & 5.860 .797 & 369.522 \\
\hline 2003 & 19.806 .960 & 12.742 .049 & 308.101 & 6.317 .293 & 439.517 \\
\hline 2004 & 20.878 .438 & 13.388 .011 & 264.585 & 6.707 .535 & 518.307 \\
\hline 2005 & 22.138 .478 & 14.300 .380 & 268.817 & 6.980 .738 & 588.543 \\
\hline 2006 & 24.907 .229 & 16.411 .813 & 310.189 & 7.462 .918 & 722.309 \\
\hline 2007 & 26.747 .197 & 17.696 .623 & 322.078 & 7.849 .491 & 879.005 \\
\hline 2008 & 29.287 .903 & 19.420 .942 & 333.287 & 8.453 .601 & 1.080 .073 \\
\hline 2009 & 30.890 .136 & 20.519 .224 & 337.465 & 8.835 .194 & 1.198 .253 \\
\hline 2010 & 31.636 .258 & 21.152 .773 & 313.984 & 9.015 .356 & 1.154 .145 \\
\hline 2011 & 33.262 .998 & 22.368 .598 & 332.578 & 9.251 .425 & 1.310 .397 \\
\hline 2012 & 34.875 .937 & 23.569 .623 & 338.520 & 9.395 .466 & 1.582 .228 \\
\hline 2013 & 36.744 .838 & 24.819 .922 & 347.144 & 9.704 .131 & 1.873 .641 \\
\hline 2014 & 38.027 .171 & 25.543 .908 & 347.720 & 9.863 .980 & 2.271 .563 \\
\hline 2015 & 39.975 .998 & 26.907 .994 & 357.452 & 10.073 .288 & 2.637 .264 \\
\hline 2016 & 42.454 .265 & 28.664 .295 & 367.772 & 10.384 .621 & 3.037 .577 \\
\hline 2017 & 45.853 .522 & 30.958 .042 & 401.120 & 10.985 .817 & 3.598 .543 \\
\hline
\end{tabular}

Fuente: elaboración propia con datos del INEGI (2017). 
la interacción cotidiana, debido principalmente a la cultura vial.

Efectivamente, el número de vehículos de motor registrados en México creció desproporcionadamente, en comparación con la población total del país. Es decir, el índice de motorización aumentó de 169 vehículos por habitante en el año 2000, a 315 por habitante en 2010 (tabla 4).

Como se observa en la figura 1, la ciudad de Tepic está ubicada en el noroeste de México, sobre el Ilamado Valle de Matatipac. Es la capital del estado de Nayarit. Es una zona metropolitana constituida por dos municipios: Tepic y Xalisco. Según el censo de población y vivienda de 2010, la población era de 332.863 habitantes y representaba el $31 \%$ del total del estado (INEGI, 2010).

Aunado a que funge como la capital del estado, se posiciona como nicho político-comercial. Las distintas etapas que pueden distinguirse del crecimiento de la ciudad se han basado en un urbanismo de acción (Ruiz, 2001, p. 54), donde la
Tabla 4. Índice de motorización en México

\begin{tabular}{cc}
\hline Año & $\begin{array}{c}\text { Automóviles por 1000 } \\
\text { habitantes }\end{array}$ \\
\hline 2000 & 169 \\
\hline 2001 & 181 \\
\hline 2002 & 196 \\
\hline 2003 & 194 \\
\hline 2004 & 203 \\
\hline 2005 & 213 \\
\hline 2006 & 237 \\
\hline 2007 & 251 \\
\hline 2008 & 273 \\
\hline 2009 & 287 \\
\hline 2010 & 315 \\
\hline 2011 & 332 \\
\hline 2012 & 349 \\
\hline 2013 & 368 \\
\hline 2014 & 380 \\
\hline 2015 & 400 \\
\hline 2016 & 424 \\
\hline 2017 & 458 \\
\hline
\end{tabular}

Fuente: elaboración propia con datos del INEGI (2017).

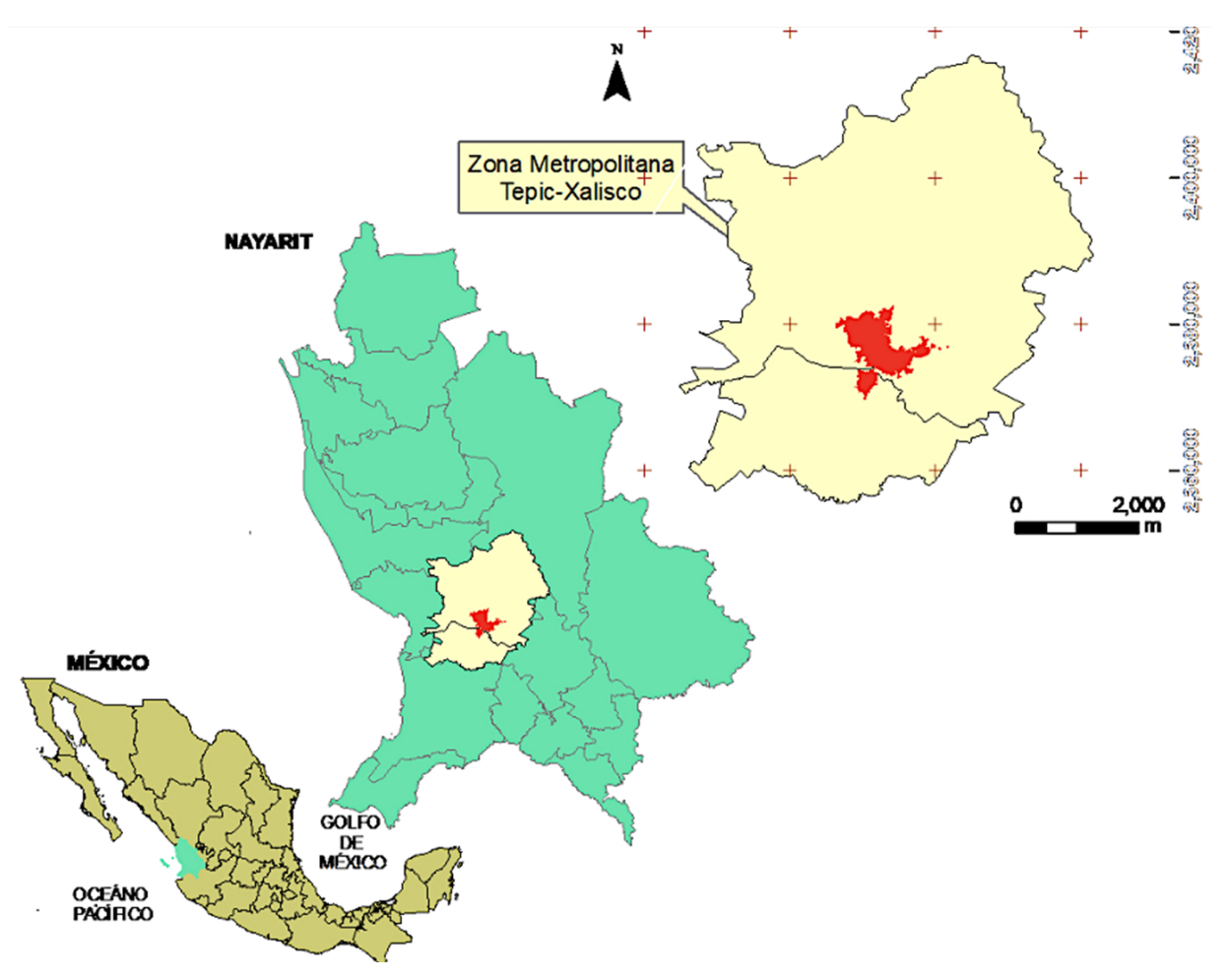

Figura 1. La ciudad de Tepic, México

Fuente: elaboración propia. 
construcción y el crecimiento de la ciudad se han dado mediante decisiones voluntaristas de quienes ostentan el poder o componen parte de él, y los planes y legislaciones dictados en materia de crecimiento urbano-habitacional han estado encaminadas a dar legalidad a estas decisiones voluntariosas. Efectivamente, la urbanización del medio rural ha permitido acceder a vivienda de interés social o popular; sin embargo, ubicadas en las periferias donde se adolece de infraestructura y equipamiento, con costos sociales significativos para la movilidad. "Este escenario coloca en desventaja al residente de esa primera o segunda periferia, que utiliza frecuentemente el servicio de transporte público" (González, 2018b, p. 67).

Actualmente la ciudad de Tepic tiene una estructura socioterritorial que constituye un continuum urbano con el municipio de Xalisco y con otras localidades que forman parte del propio municipio de Tepic, lo que evidencia una creciente concentración de población, servicios, poder político y económico. En la tabla 5 se pueden observar las fases de crecimiento que ha tenido la ciudad desde los años 1970 hasta la actualidad.

\section{METODOLOGÍA}

Para estimar las emisiones de gases de efecto invernadero (GEI) para el año base por cada modo de transporte en un año determinado, se calcula el consumo de energía por modo y tipo de combustible, y se utilizan los factores de emisión proporcionados por el IPCC. En este caso se usan factores de emisión de la metodología de 1996, dado que es la última aprobada por la convención de Cambio Climático de las Naciones Unidas (2018).
Para calcular el consumo de energía del transporte de pasajeros se utiliza la ecuación (1).

$$
E_{t o}=\sum_{i j k}\left(V_{i j k} R_{i j k} D_{i} A_{j} P c_{k}\right)
$$

Donde:

$E_{\text {to }}=$ consumo de energía del transporte de pasajeros del año base.

$V=$ cantidad de vehículos de pasajeros de la zona en cuestión.

$R=$ rendimiento de combustible (litros $/ \mathrm{km}$ ).

$D=$ distancia recorrida por vehículo al día $(\mathrm{km})$.

$A=$ días en circulación al año.

$P_{C}=$ poder calorífico del combustible (julios/litro).

$i=$ tipo de vehículo.

$j=$ año del modelo del vehículo.

$k=$ tipo de combustible.

En este trabajo se analizó únicamente el transporte de pasajeros tipo autobuses de la ciudad de Tepic, capital del estado de Nayarit, en base a la información proporcionada por la Secretaría del Medio Ambiente de Nayarit (Semanay). En este entendido, conforme a la metodología del IPCC, las emisiones de dióxido de carbono $\left(\mathrm{CO}_{2}\right)$ se calcularon de acuerdo con la ecuación (2).

$$
\text { Emisiones } \mathrm{CO}_{2}=\sum_{k}\left(C_{k} F e_{k} F O_{k}\right) * \frac{44}{12}
$$

Donde:

$C=$ combustible consumido $k$.

$F e=$ factor de emisión para el combustible $k$.

$F o=$ fracción oxidada para el combustible $k$.

$k=$ tipo de combustible.

Tanto las fracciones de oxidación como los factores de emisión se muestran en la tabla 6 .

Tabla 5. Población total del municipio de Tepic y la ciudad de Tepic

\begin{tabular}{llllll}
\hline \multicolumn{1}{c}{ Año } & \multicolumn{1}{c}{$\mathbf{1 9 7 0}$} & $\mathbf{1 9 8 0}$ & $\mathbf{1 9 9 0}$ & \multicolumn{1}{c}{$\mathbf{2 0 0 0}$} & \multicolumn{1}{c}{$\mathbf{2 0 1 0}$} \\
\hline Municipio Tepic & 110.939 & 177.007 & 241.463 & 305.176 & 380.249 \\
\hline Ciudad Tepic & No disponible & No disponible & 206.967 & 265.817 & 332.863 \\
\hline
\end{tabular}

Fuente: elaboración propia con datos de INEGI (2010). 
Tabla 6. Emisiones de $\mathrm{CO}_{2}$

\begin{tabular}{lccc}
\hline \multicolumn{2}{c}{ Fracción de carbono oxidada } & \multicolumn{2}{c}{ Factor de emisión $\mathbf{C O}_{\mathbf{2}}$} \\
\hline Carbón & 0,980 & GLP & 62,436 \\
\hline Petróleo y productos & 0,990 & Gasolinas & 68,607 \\
\hline Gas & 0,995 & Diésel & 73,326 \\
\hline Peat para generación eléctrica & 0,990 & Gas natural & 55,82 \\
\hline
\end{tabular}

Fuente: IPCC (2017).

\section{RESULTADOS}

El sistema de transporte público de la ciudad de Tepic es en su totalidad prestado por el sector privado. La mayoría de la movilidad urbana se efectúa en camiones (autobuses) y combis. En la tabla 7 se presenta un resumen de los diferentes tipos de transporte público.
De acuerdo con la metodología aplicada, para estimar el consumo de combustibles es necesario conocer el recorrido anual por tipo de vehículo, así como su rendimiento vehicular. La tabla 8 muestra las distancias recorridas en kilómetros en periodos distintos, y la tabla 9 indica la estimación promedio del rendimiento vehicular por tipo de vehículo.

Tabla 7. Número de unidades y rutas de transporte en Tepic

\begin{tabular}{lcc}
\hline \multicolumn{1}{c}{ Tipo } & Cantidad & Rutas \\
\hline Camión (buses/busetas)) & 574 & 38 \\
\hline Combis (colectivos) & 235 & 30 \\
\hline Taxis & 4559 & $\mathrm{NA}$ \\
\hline Camiones de carga & 208 & $\mathrm{NA}$ \\
\hline Total & 5576 & \\
\hline
\end{tabular}

NA: no aplica.

Fuente: Dirección General de Tránsito y Transporte de Nayarit (2012).

Tabla 8. Kilómetros recorridos por tipo de vehículo y periodo en la ciudad de Tepic

\begin{tabular}{lrrrr}
\hline \multirow{2}{*}{ Tipo de vehículo } & \multicolumn{4}{c}{ Kilómetros recorridos } \\
\cline { 2 - 5 } & Día & Semana & Mes & Año \\
\hline Combis (colectivos) & 296 & 2072 & 8878 & 108017 \\
\hline Vehículos $<3$ ton & 273 & 1908 & 8177 & 99482 \\
\hline Vehículos $>3$ ton & 229 & 1600 & 6857 & 83.429 \\
\hline Taxis & 206 & 1442 & 6178 & 75.164 \\
\hline Camiones (buses/busetas) & 205 & 1437 & 6161 & 74.953 \\
\hline Automóvil & 36 & 250 & 1072 & 13.039 \\
\hline Pick-up & 36 & 250 & 1069 & 13.010 \\
\hline Camionetas (SUV) & 33 & 229 & 982 & 11.952 \\
\hline Motocicletas & 18 & 129 & 554 & 6738 \\
\hline
\end{tabular}

Fuente: Comisión para la Cooperación Ambiental (2009). 
Las emisiones de GEI de la ciudad de Tepic fueron de casi 192.000 toneladas emitidas solo por el transporte público de la ciudad de Tepic, en donde se puede observar que las fuentes que más contaminan son las unidades tipo camión (autobuses de pasajeros), tal y como se muestra en la tabla 10.

Aunque el otorgamiento de concesiones de transporte público se ha venido dando de manera desordenada y arbitraria por parte de los gobiernos, se puede crear un escenario de prospectivo del parque vehicular al 2020 para la ciudad de
Tepic. Así, el parque vehicular quedaría de acuerdo con los datos que se detallan en la tabla 11.

Estos datos muestran el aumento que podría tener el parque vehicular de seguir con esta tendencia de crecimiento. Las afectaciones se concentrarían en la eficiencia de los traslados cotidianos, el consumo de combustible, lo que traería como consecuencia un crecimiento en las emisiones de GEI impactando de manera negativa en la salud de los habitantes. Por ello, un programa de mitigación ante este escenario podría acentuar el

Tabla 9. Rendimiento combustible por tipo de vehículo

\begin{tabular}{lcc}
\hline \multirow{2}{*}{\multicolumn{1}{c}{ Tipo de vehículo }} & Tipo & Rendimiento \\
\cline { 2 - 3 } & combustible & $\mathbf{k m} / \mathbf{l}$ \\
\hline Subcompactos & Gasolina & 10 \\
\hline Lujo y usos múltiples & Gasolina & 7 \\
\hline Deportivos & Gasolina & 4 \\
\hline Taxis & Gasolina & 10 \\
\hline Microbús & Gasolina & 2 \\
\hline Motocicletas & Gasolina & 15 \\
\hline Camiones (autobuses de pasajeros) & Diésel & 1,8 \\
\hline
\end{tabular}

Fuente: elaboración propia con datos de Sederma (2017).

Tabla 10. Emisiones de GEI del transporte público de la ciudad de Tepic

\begin{tabular}{lcc}
\hline \multicolumn{1}{c}{ Tipo de transporte } & $\begin{array}{c}\text { Miles de } \\
\text { toneladas de } \mathbf{C O}_{\mathbf{2}}\end{array}$ & $\mathbf{\%}$ \\
\hline Taxi & 39 & 20,31 \\
\hline Combis (colectivos) & 41 & 21,35 \\
\hline Camión (buses/busetas) & 112 & 58,33 \\
\hline Total & 192 & 100,00 \\
\hline
\end{tabular}

Fuente: elaboración propia.

Tabla 11. Escenario prospectivo del parque vehicular a 2020

\begin{tabular}{lcc}
\hline \multicolumn{1}{c}{ Tipo } & $\mathbf{2 0 1 5}$ & $\mathbf{2 0 2 0}$ \\
\hline Camión (buses/busetas) & 574 & 955 \\
\hline Combis (colectivos) & 235 & 946 \\
\hline Taxis & 4559 & 9570 \\
\hline Camiones de carga & 208 & 385 \\
\hline Total & 5576 & 11.856 \\
\hline
\end{tabular}

Fuente: elaboración propia. 
denominado "Hoy no Circula", el cual se ha implementado desde hace varios años en la ciudad de México para regular el uso del vehículo privado, con efectos cuestionables en materia de emisión de gases; sin embargo, sus objetivos se han centrado en la concientización del uso racional del vehículo e incentivar el uso del transporte público y colectivo.

\section{CONCLUSIONES}

De continuar las tendencias actuales en el crecimiento de parques vehiculares particulares, sumado al consumo energético y emisiones de GEI de un transporte público obsoleto, la ciudad estaría comprometiendo la calidad de su aire. En este sentido, el incremento vehicular, carente de planificación, no prevé escenarios relativos al congestionamiento vial y la reducción de las velocidades de circulación y los tiempos de traslado en las principales avenidas de la ciudad. Actualmente, estas presentan serios congestionamientos en determinados horarios y días de la semana que afectan el rendimiento promedio de combustible por unidad. En este sentido, no solo es conveniente sino necesario que las autoridades del estado inicien transformaciones integrales a la política pública de transporte, tendiente a mitigar las externalidades del modo de desplazarse en la ciudad. Ello implica, un análisis profundo de las fuentes de energía utilizadas, la promoción del vehículo privado y la omisión de un transporte público viejo y en mal estado.

\section{REFERENCIAS}

Alfie, M. (2007). Una nueva gestión ambiental: El riesgo y el principio precautorio. Espacio Abierto, 16(2), 209-222. Recuperado de https://www.redalyc.org/ articulo.oa?id=12216202

Arizaga B., A., González P., M.G. y Asprilla L., Y. (2019). Sistemas piezoeléctricos en el tren urbano de Guadalajara, México: entropía y negentropía. Tecnura, 23(61), 13-22. https://doi. org/10.14483/22487638.14870
Comisión para la Cooperación Ambiental (2009). Medición y adaptación de variables de actividad vehicular en ciudades tipo de México. Recuperado de http://www3.cec.org/islandora/es/item/3696-measurement-and-adaptation-vehicle-activity-variables-in-mexican-sample-cities-summary-es

Dirección General de Tránsito y Transporte de Nayarit (2012). No. de oficio DGTT/DG/0763/07/2012. Asunto contestación de oficio. Nayarit.

García de Quevedo, N.F., González P., M.G. y Asprilla L., Y. (2018). Determinación de los componentes entrópicos de la accidentalidad: el trinomio vehículo/usuario/camino en la metrópoli de Guadalajara, México. Tecnura, 22(55), 51-65. https://doi. org/10.14483/22487638.13245

González Pérez, M.G. (2018a). Entropy and negentropy of private electric vehicles in urban systems: homeostasis of mobility in Mexico. DYNA, 85(206), 171-177. https://doi.org/10.15446/dyna. v85n206.72509

González Pérez, M.G. (2018b). Movilidad cotidiana y habitar periurbano en el área metropolitana de Guadalajara: entre el costo y el beneficio social. Contexto, 12(16), 67-77. Recuperado de http://contexto.uanl.mx/index.php/contexto/article/view/144 Instituto Nacional de Estadística y Geografía (INEGI). (2010). Censo de Población y Vivienda 2010. Recuperado de https://www.inegi.org.mx/programas/ ccpv/2010/

Instituto Nacional de Estadística y Geografía (INEGI) (2017). Banco de Información Económica (BIE). Recuperado de www.inegi.org.mx/sistemas/bie/

Nakicenovic, N. (2000). Greenhouse gas emissions scenarios. Technological Forecasting \& Social Chance, 2(65), 149-166. https://doi.org/10.1016/ S0040-1625(00)00094-9

Organización de Naciones Unidas (ONU) (2018). Cambio climático. Recuperado de https://www.un.org/es/ sections/issues-depth/climate-change/index.html

Panel Intergubernamental del Cambio Climático (IPCC) (2017). Cambio climático 2014. Impactos, adaptación y vulnerabilidad. Recuperado de https:// archive.ipcc.ch/pdf/assessment-report/ar5/wg2/ WGIIAR5-IntegrationBrochure_es.pdf 
Ruiz J. (2001). Sistemas urbanos complejos acción y comunicación. Cuadernos de Investigación Urbanística, 32(1), 1-78. Recuperado de http://polired.upm. es/index.php/ciur/article/view/246/242

Secretaría del Medio Ambiente y Recursos Naturales (Semarnat) (2018). Registro Nacional de Emisiones. Recuperado de https://www. gob.mx/semarnat/acciones-y-programas/ registro-nacional-de-emisiones-rene
Secretaría de Desarrollo Rural y Medio Ambiente (Sederma) (2017). Sistema Nacional de Información de la Calidad del Aire (Sinaica). Recuperado de https://sinaica.inecc.gob.mx/archivo/informes/Informe2017.pdf

Secretaria de Energía (Sener) (2018). Balance Nacional de Energía 2017. México. Recuperado de https://www. gob.mx/cms/uploads/attachment/file/414843/Balance_Nacional_de_Energ_a_2017.pdf

\section{(c) (i) (2)

